\title{
Unmarked gene modification in Streptococcus mutans by a cotransformation strategy with a thermosensitive plasmid
}

\author{
Indranil Biswas, Laura Drake, Sean Johnson, and Damon Thielen \\ Sanford School of Medicine, University of South Dakota, Vermillion, SD, USA \\ BioTechniques 42:487-490 (April 2007) \\ doi 10.2144/000112414
}

Unmarked gene modifications are desirable for various genetic analyses; however, they have been difficult to construct without selectable markers. We describe here a new genetic method for constructing unmarked mutants in Streptococcus mutans. The desired mutant allele is first constructed and introduced into the strain by cotransformation with pGhost 4 , a thermosensitive plasmid that replicates in several low $G+C$ Gram positive bacteria. With this method, we have modified two different loci with high frequency by insertion or deletion in S. mutans. Because pGhost 4 contains a broad host range thermosensitive replicon, this method can be applied to any transformable low $G+C$ Gram positive bacteria, including oral streptococci.

\section{INTRODUCTION}

To understand the putative function of a gene, one needs to either inactivate or selectively modify it. In vitro transposition mutagenesis and gene disruption by homologous recombination using an antibiotic resistance cassette are the most common techniques for gene inactivation. The classical strategy for gene disruption requires cloning of the gene to be mutated, insertional inactivation of the gene with an antibiotic resistance cassette, and exchange of the plasmid-borne mutant allele with the bacterial chromosome. PCR and other recombinational technologies can also be exploited to substantially accelerate virtually all steps involved in the gene disruption process. However, all these methods require utilization of an antibiotic resistance marker, which often causes polar effect on the downstream genes. Moreover, these disruption procedures cannot be used for insertion or deletion of single amino acids or modification of single or multiple amino acids. These modifications are important to study biological functions of a given protein.

The use of a thermosensitive plasmid such as pGhost4 (1) in gene disruption can circumvent the use of antibiotic resistance cassette for unmarked gene modification; however this method is labor-intensive and time-consuming (2). Genetic analysis of many oral streptococci, including Streptococcus mutans, has thus far been limited in scope due to the lack of advanced genetic tools. Although some basic tools that work in other low $\mathrm{G}+\mathrm{C}$ Gram positive bacteria can be used in oral streptococci, their effectiveness is very much limited. Here we develop a rapid and easy mutagenesis strategy for $S$. mutans without the use of selectable markers. This strategy exploits the fact that $S$. mutans is competent for genetic transformation and can be cotransformed (i.e., can uptake two different DNA molecules by a competent cell effectively). We show that this method is highly efficient for insertion or deletion modification of chromosomal genes.

\section{MATERIALS AND METHODS}

\section{Bacterial Strains and Growth Conditions}

Escherichia coli strain DH5 $\alpha$ and TG1Rep+ (3) were grown in LB medium. TG1Rep+ contains a chromosomal copy of the wild-type repA gene and was used as a host for propagation of pGhost 4 at $37^{\circ} \mathrm{C}$. Thereafter, $100 \mu \mathrm{g} /$
$\mathrm{mL}$ ampicillin, $100 \mu \mathrm{g} / \mathrm{mL}$ kanamycin, $100 \mu \mathrm{g} / \mathrm{mL}$ spectinomycin, and/or 250 $\mu \mathrm{g} / \mathrm{mL}$ erythromycin were added to the LB when needed. $S$. mutans strain UA159 was grown in Todd-Hewitt broth with $0.2 \%$ yeast extract (THY), and when necessary, $300 \mu \mathrm{g} / \mathrm{mL}$ kanamycin, $300 \mu \mathrm{g} / \mathrm{mL}$ spectinomycin, and/or $10 \mu \mathrm{g} / \mathrm{mL}$ erythromycin were added.

\section{Transformation of $S$. mutans and Screening of Mutants}

S. mutans cultures for transformations were grown in THY with 5\% heat-inactivated horse serum. Bacterial cultures were grown at $37^{\circ} \mathrm{C}$ until the absorbance reached at $600 \mathrm{~nm}$ was approximately 0.2 . At this point, competence-stimulating peptide (CSP) was added at a final concentration of $500 \mathrm{ng} /$ $\mathrm{mL}$. The amino acid sequence of CSP is SGSLSTFFRLFNRSFTQALGK (4), and it was synthesized by Mimotopes Pvt. Ltd (Clayton, VIC, Australia). Transforming DNA was added to the culture and incubated for $60-90 \mathrm{~min}$ at $30^{\circ} \mathrm{C}$. We routinely used $200 \mathrm{ng} / \mathrm{mL}$ plasmid DNA with or without $1 \mu \mathrm{g} / \mathrm{mL}$ second unselected target locus DNA. The transformed cells were plated on antibiotic-containing media and further incubated for $48 \mathrm{~h}$ at $30^{\circ} \mathrm{C}$ under microaerophilic conditions. Transformants that appeared on the selective media were checked for unselected chromosomal recombination events (Figure 1) either by patching on antibiotic plates or by quick lysis followed by PCR verification. Quick lysis PCR was performed using PrepMan ${ }^{\circledR}$ Ultra reagents (Applied Biosystems, Foster City, CA, USA) following the manufacturer's instructions. A small bacterial colony was resuspended in PrepMan Ultra reagent and heated for $10 \mathrm{~min}$ at $100^{\circ} \mathrm{C}$. PCR was performed using an aliquot of this sample after removal of cell debris from lysed bacteria by centrifugation.

\section{Plasmid and DNA Constructs}

Plasmid pGhost 4 is derived from the broad host range replicon pWV01 and contains a thermosensitive origin of replication (due to mutations in the repA gene, rep $A^{\mathrm{Ts}}$ ) and an eryth- 
romycin resistance $\left(\mathrm{Em}^{\mathrm{R}}\right)$ marker (1). For modification of htrA locus, plasmid pIB102 was used as described previously (5). To inactivate $S m u 486$ locus, two separate constructs were made. First, a 1.47-kb fragment spanning the entire Smu486 region was amplified by PCR from UA159 genomic DNA using primers BamSmu486-F1 (5'-cgcggatccTGTTGAT GTCTCAGTTAGTTTGG-3') and Bam-Smu487-R1 (5'-cgcggatccTT TAGCAACCTGCTTCAATG ACAGG$3^{\prime}$ ), in which the lowercase sequences denote the incorporated restriction sites. This fragment was cloned into $\mathrm{pGEM}^{{ }^{\circledR}}$ T-Easy vector (Promega, Madison, WI, USA) to create pIB38. A $0.87-\mathrm{kb}$ spectinomycin resistance gene (aad 9$)$ was amplified from pUCSpec (6) using primers Spec-P-For (5'-TATCTAGAAT AAAAAATTTAGAAGCCCAATG-3') and Spec-Rev (5'-TTTTATAATTTTT TTAATCTGTTATTTAAATAG- $3^{\prime}$ ) and cloned into XhoI-XbaI-digested and T4 polymerase-blunted pIB38 to generate pIB74. In this plasmid, a $0.37-\mathrm{kb}$ internal region of $5 m u 486$ is replaced with the aad 9 gene. To generate pIB45, pIB38 was digested with XhoI and $X b a \mathrm{I}$ and blunt-ended with $\mathrm{T} 4$ polymerase followed by self-ligation. Thus, in pIB 45, the same $0.37-\mathrm{kb}$ region of Smu 486 is deleted.

Plasmids pIB45, pIB74, and pIB102 were used as template for PCR amplification to generate linear fragment using M13 forward and reverse primers [sequence present in the multiple cloning site (MCS) in the vector plasmids]. The PCR fragments were quantitated and used for transformation at the required amount.

\section{RESULTS AND DISCUSSION}

S. mutans can achieve a moderate level of competence compared with highly competent streptococci such as Streptococcus pneumoniae $(4,7)$. To verify whether the cotransformation can be successfully performed in S. mutans, two DNA molecules, a circular thermosensitive plasmid pGhost 4 , which has a broad host range, and a linear DNA molecule, were used. The pGhost4 plasmid was used for selection purpose, whereas the linear DNA molecule

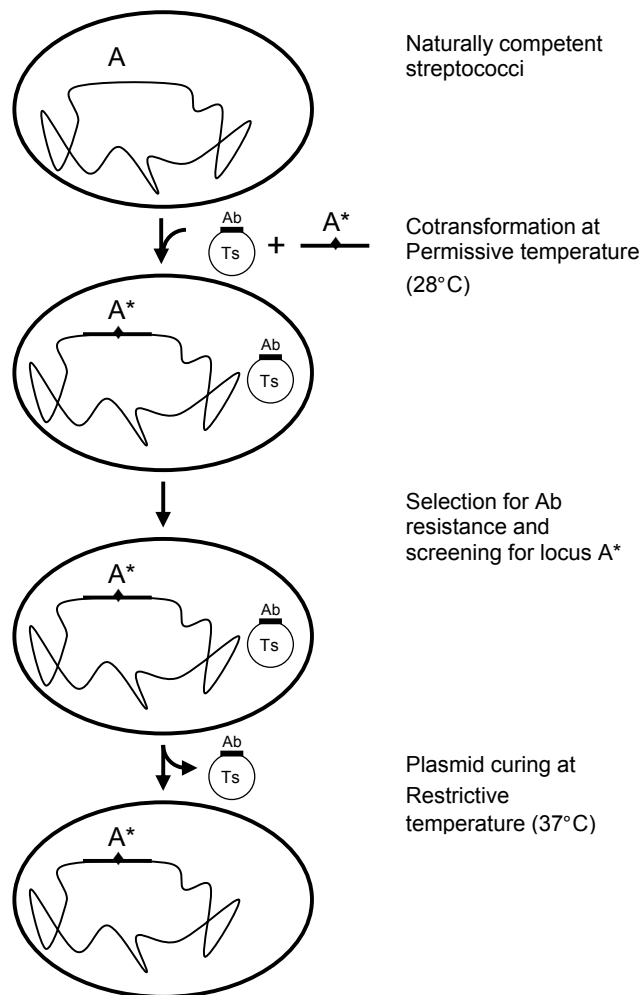

Figure 1. Diagram for markerless genetic exchange based on cotransformation. A linear chromosomal DNA fragment containing a desired mutation (indicated as $\mathrm{A}^{*}$ ) is transformed along with a thermosensitive $\mathrm{pG}$ host 4 plasmid and selected for plasmid-borne marker. The desired mutation is identified among the transformed bacteria by PCR or a detectable phenotype. The pGhost 4 plasmid is cured from the selected strain by growing at restrictive temperature. $\mathrm{Ab}$, antibiotic resistance marker; Ts, thermosensitive.

served as target (unselected) locus. Among the transformants, targeted loci were screened using the corresponding antibiotic resistance marker they carry.

A ratio of 1:5 of selected versus targeted molecules was initially chosen for transformation and plated on the selective media specific for the selected marker. Figure 1 shows the overall strategy for this method. The target loci that were selected for this purpose are htrA and Smu486. For faster screening,

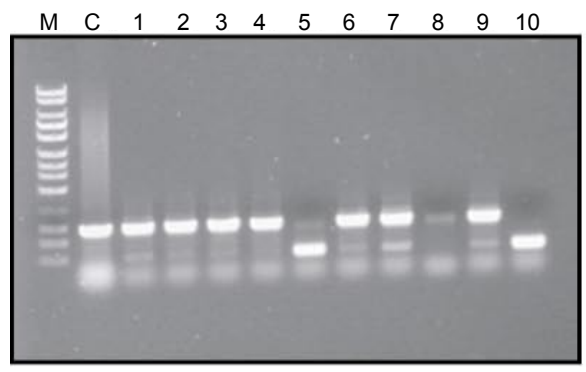

Figure 2. PCR screening of the transformants from unmarked deletion mutations at Smu486 locus. Templates were made from 10 randomly chosen transformants using PrepMan Ultra reagent. M, 1-kb DNA ladder (Thermo Fisher Scientific, Waltham, MA, USA); C, wildtype control strain; lanes 1-10, samples from 10 clones. Note that lanes 5 and 10 contain the unmarked deletion mutation. 
Table 1. Efficiency of Unmarked Gene Modification in Streptococcus mutans

\begin{tabular}{|c|c|c|c|}
\hline Transforming DNA & $\begin{array}{l}\text { Target Mutation } \\
\text { (Homology) }^{\mathrm{a}}\end{array}$ & Frequencyb & Efficiencyc \\
\hline pGhost4d & N.A. & $(3.5 \pm 2.4) \times 10^{-6}$ & N.A. \\
\hline$h t r A^{d}$ & Insertion (1.08/0.85) & $(3.1 \pm 2.0) \times 10^{-5}$ & N.A. \\
\hline Smu486d & Insertion $(0.58 / 0.52)$ & $(6.6 \pm 5.4) \times 10^{-3}$ & N.A. \\
\hline pGhost4 + htrA & Insertion (1.08/0.85) & $\begin{array}{l}(2.0 \pm 1.1) \times 10^{-6}(\text { pGhost } 4) \\
\left(1.9 \pm 1.9 \times 10^{-6}(\text { htrA })\right.\end{array}$ & $73 \% \pm 27 \%$ \\
\hline pGhost4 + Smu486 & Insertion (0.58/0.52) & $\begin{array}{l}(1.1 \pm 0.2) \times 10^{-6} \quad(\text { pGhost } 4) \\
\left(5.4 \pm 0.7 \times 10^{-4} \quad(\text { Smu486) }\right.\end{array}$ & $18 \% \pm 1 \%$ \\
\hline pGhost4 + Smu486 & Deletion (0.58/0.52) & $\begin{array}{l}(2.0 \pm 1.1) \times 10^{-6}(\text { pGhost } 4) \\
\text { Unselected }\end{array}$ & $10 \% \pm 2 \%$ \\
\hline \multicolumn{4}{|c|}{$\begin{array}{l}\text { N.A., not applicable. } \\
\text { aLength of homology (in kb) at the left and right flanking region of the insertion or deletion. } \\
\text { bFrequency of transformation was obtained by dividing the number of colonies on the media containing } \\
\text { antibiotics by the number of colonies on media without any antibiotics. Values are the mean and stan- } \\
\text { dard error of at least two independent transformation experiments using either the target locus alone (or } \\
\text { pGhost } 4 \text { alone) or pGhost } 4 \text { in combination with the target locus. In case of cotransformation, selected } \\
\text { loci are indicated in parenthesis. } \\
\text { 'Calculated as the number of colonies containing the chromosomal modification divided by the number of } \\
\text { erythromycin resistance (Em) colonies (pGhost } 4 \text { ) examined. Shown are the mean and standard error of } \\
\text { at least two independent experiments. } \\
\text { dControl transformations were done for obtaining transformation frequency of single locus. }\end{array}$} \\
\hline
\end{tabular}

PCR, as described in the Materials and Methods section. Among the $\mathrm{Em}^{\mathrm{R}}$ colonies, about $10 \%$ colonies also showed a second recombination event at the Smu486 locus (Figure 2). The efficiency of second recombination event appears to be lower in the deletion modification compared with the insertion modification (10\% versus $18 \%$ ). It is possible that the longer DNA fragment (insertion construct) is taken up more efficiently than the smaller DNA fragment (deletion construct). Alternatively, double crossover events favor comparable lengths between foreign and target DNA.

Once the unmarked chromosomal recombination has been identified, the pGhost 4 plasmid was then cured from the selected strains. Two such colonies were chosen, and they were grown from a single colony in $5 \mathrm{~mL}$ THY broth at $37^{\circ} \mathrm{C}$ (nonpermissive for pGhost 4 replication) without any antibiotic selection. After a single overnight growth, about $10 \%$ colonies showed erythromycin sensitive $\left(\mathrm{Em}^{\mathrm{S}}\right)$ phenotype, indicating that these strains have lost the pGhost 4 plasmid. After three successive overnight growths at high temperature without selection, over $80 \% \mathrm{Em}^{\mathrm{s}}$ colonies were obtained. The targeted modified loci were verified again by PCR in the final pGhost4-cured colonies. Thus, our results showed that by using a thermosensitive plasmid, unmarked gene modification can be efficiently performed in S. mutans.

In summary, a strategy for gene modification without antibiotic marker selection has been demonstrated to perform efficiently in S. mutans. This method requires simultaneous transformation of pGhost 4 , a thermosensitive plasmid, along with the target locus DNA fragment carrying desired modification. The transformants are first selected for the pGhost 4 marker at $30^{\circ} \mathrm{C}$, a permissive temperature for pGhost4. Desired unselected recombination events are then screened among the transformants either by PCR or by other phenotypes. Once the desired unselected mutations are found, pGhost 4 is then cured from the strain by growing at $37^{\circ} \mathrm{C}$, a nonpermissive temperature for pGhost 4 replication. This method has been successfully used to achieve both an insertion as large as $2 \mathrm{~kb}(h t r A)$ and a deletion as large as $0.4 \mathrm{~kb}$ (Smu486) in S. mutans.

The traditional use of thermosensitive plasmids for unmarked gene modification is very time-consuming, because it involves cloning of the modified fragment into the plasmid, followed by integration and excision of the thermosensitive plasmid from the chromosome $(2,8)$. The method described here is straightforward and relatively fast, and the entire method can be completed in a week. If the gene of interest has a distinct phenotype, such as clumping, capsules, and/or pigment production, mutagenesis can be achieved even faster. Several factors may influence the efficiency of this method, such as the molar ratio of the two cotransforming molecules, the length of the homology of the flanking regions, and chromosomal locus. If the recombination frequency of the target locus is low, efficiency can be improved simply by increasing the length of the homologous DNA fragment. By combining the rapid fusion PCR-mediated manipulation (9) and cotransformation-mediated unmarked gene modification, it is possible to obtain an inframe deletion and insertion, to add an epitope tag to any gene either $\mathrm{N}$ or $\mathrm{C}$ terminus, and to change a single amino acid without a laborious cloning procedure. Several streptococci are highly competent (10), and plasmid pGhost4 and its derivatives have a broad-host range thermosensitive replicon (1). Therefore, this method can be successfully used in those streptococci and possibly to other low $\mathrm{G}+\mathrm{C}$ Gram positive bacteria.

\section{ACKNOWLEDGMENTS}

We thank Dr. Maguin [Institut National de la Recherche Agronomique (INRA), France] for providing the $p G$ host 4 plasmid. This publication was made possible in part by National Institutes of Health (NIH) grant (1R21DE016056) from the National Institute of Dental and Craniofacial Research (NIDCR) awarded to I.B.

\section{COMPETING INTERESTS STATEMENT}

The authors declare no competing interests.

\section{REFERENCES}

1. Maguin, E., P. Duwat, T. Hege, D. Ehrlich, and A. Gruss. 1992. New thermosensi- 


\section{Short Technical Reports}

tive plasmid for gram-positive bacteria. J. Bacteriol. 174:5633-5638.

2. Biswas, I., A. Gruss, S.D. Ehrlich, and E. Maguin. 1993. High-efficiency gene inactivation and replacement system for gram-positive bacteria. J. Bacteriol. 175:3628-3635.

3. Buist, G., J. Kok, K.J. Leenhouts, M. Dabrowska, G. Venema, and A.J. Haandrikman. 1995. Molecular cloning and nucleotide sequence of the gene encoding the major peptidoglycan hydrolase of Lactococcus lactis, a muramidase needed for cell separation. J. Bacteriol. 177:1554-1563.

4. Cvitkovitch, D.G. 2001. Genetics competence and transformation in oral streptococci. Crit. Rev. Oral Biol. Med. 12:217-243.

5. Biswas, S. and I. Biswas. 2005. Role of HtrA in surface protein expression and biofilm formation by Streptococcus mutans. Infect. Immun. 73:6923-6934.

6. Husmann, L.K., J.R. Scott, G. Lindahl, and L. Stenberg. 1995. Expression of the Arp protein, a member of the M protein family, is not sufficient to inhibit phagocytosis of Streptococcus pyogenes. Infect. Immun. 63:345-348.

7. Iannelli, F. and G. Pozzi. 2004. Methods for introducing specific and unmarked mutations into the chromosome of Streptococcus pneumoniae. Mol. Biotechnol. 26:81-86.

8. Atlagic, D., A.O. Kilic, and L. Tao. 2006. Unmarked gene deletion mutagenesis of $g t f B$ and gtfC in Streptococcus mutans using a targeted hit-and-run strategy with a thermosensitive plasmid. Oral Microbiol. Immunol. 21:132-135.

9. Horton, R.M. 1995. PCR-mediated recombination and mutagenesis. SOEing together tailor-made genes. Mol. Biotechnol. 3:93-99.

10. Havarstein, L., R. Hakenbeck, and $\mathbf{P}$. Gaustad. 1997. Natural competence in the genus Streptococus: evidence that streptococci can change pherotype by interspecies recombinational exchanges. J. Bacteriol. 179:6589-6594.

Received 13 July 2006; accepted 2 January 2007.

Address correspondence to Indranil Biswas, Division of Basic Biomedical Sciences, University of South Dakota Sanford School of Medicine, Lee Medical Building, 414 East Clark Street, Vermillion, SD 57069, USA.e-mail: ibiswas@usd.edu

To purchase reprints of this article, contact: Reprints@BioTechniques.com 